\title{
Experimental Study on Vibration System of Vehicle Engines Based on
}

\section{Magneto Rheological Mounts}

\author{
Zuo-yue Chen ${ }^{1, \text { a }}$ \\ ${ }^{1}$ Department of Electromechanical Engineering, Leshan Vocational and Technical College, Leshan, \\ Sichuan, China, 614000 \\ a email
}

Keywords: Magneto rheological Mount, Vibration Isolation, Dynamic Stiffness, Damping Angel

\begin{abstract}
The active vibration isolation of the engine in the range of broad frequency can realize by the controllable damping characteristics of the magneto rheological damper. This paper firstly discusses the principle of the experiment and then explores the equipment and process of the experiment of vibration control of magneto rheological mounts of vehicle engines. The results indicate that magneto- rheological semi- active mount had better performance of vibration isolation can improve vehicle ride comfort.
\end{abstract}

\section{Introduction}

The engine mount is an elastic connecting element between the engine and the frame and the body, which is used to effectively isolate the bidirectional vibration caused by the road roughness input and the engine excitation. Its performance directly affects the ride comfort of the vehicle and the working environment and service life of the components. With the wide use of the four cylinder engine with the lighter weight and balance of the car, the vibration isolation performance of the automotive suspension system is more and more high. The ideal engine mount system and $\mathrm{L}$ should have high damping characteristics in low frequency and large amplitude vibration. In order to weaken the pavement to transfer the vibration of the engine; and in high frequency and small amplitude vibration should have small stiffness and damping characteristics. In order to reduce the impact of engine vibration on the body, it is required that the stiffness and damping of the suspension has a non-linear characteristic that varies with the change of frequency and amplitude. At present, the general use of the vehicle is rubber mount and ordinary hydraulic mount. It is found that the rubber mount has better vibration isolation performance only in the low frequency band, and the vibration effect becomes poor at high frequency. This paper is the development of magneto rheological suspension as the object, after the preliminary structure design, based on the fluid mechanics theory, a mathematical model of the magneto rheological suspension, the dynamic characteristics simulation, by adjusting the structure parameters, obtained good simulation results.

\section{Principle of the Vibration System Experiment of Vehicle Engines}

The dynamic characteristics of the vibration isolation system are directly related to the vibration isolation effect of the system. Therefore, the measurement of the dynamic characteristics is helpful to understand the performance of the components and to provide guidance for the design of the components. Experimental process is generally done by dynamic testing machine. The principle is basically by processing the measured force signal and displacement signal method to get the dynamic characteristics of components. In the above method, there are two kinds of choices in the 
position of the force sensor. The first is to install it at the input end, measured by the sensor input force signal and displacement signal to get the dynamic characteristics of the test components, known as the origin of dynamic characteristics. But this method has a drawback, is the sensor in the process of movement and the upper end of the actuator to move together, therefore, the measured force including the inertia force of some other quality, the measurement and calculation process of trouble. The other is to install it at the output end, the sensor measured by the transfer force signal and displacement signal to get the dynamic characteristics of the test components, known as cross dynamic characteristics. The advantage of this method is that it does not have to consider the impact of the additional mass, so the test process is relatively simple, and the sensor is easy to install. In practical measurement, the method of measuring the dynamic characteristics of the cross point is often used, which indicates that the parameters of the dynamic characteristics of the cross point have the dynamic stiffness and the damping lag angle. Under the excitations of the sinusoidal signal $X_{m}=\bar{X}_{m} \sin 2 \pi f t$, the crossing dynamic stiffness can be calculated as the following formula.

$$
K^{*}\left(w, X_{m}\right)=K\left(w, X_{m}\right) e^{j \phi\left(w, X_{m}\right)}
$$

$K^{*}\left(w, X_{m}\right)$ is the ratio of the amplitude of the first harmonic and the excitation displacement signal of the dynamic stiffness; $K\left(w, X_{m}\right)$ is the in-phase dynamic stiffness, which is characterized by its stiffness.

\section{Process of the Vibration System Experiment of Vehicle Engines}

In order to validate the performance of simulation model and dynamic characteristics of the suspension of the sample, this paper carried out the dynamic experiment. The main purpose is to obtain the sample mount dynamic stiffness and damping angle curve. We need a direct current regulated power supply, which is used to accurately enter the corresponding control current. The external input current can obviously change the magnetic field strength of the internal controllable channel, and then change the properties of the magneto rheological fluid which flows through the controllable channel, thereby changing the dynamic stiffness and damping of the suspension. So in this experiment, the sensitive factors were analyzed. The excitation is simulated by the motor, coupling, gearbox, eccentric block, frequency converter. Engine excitation force is realized by rotating the eccentric block simulation work, the motor drives the gear box gear to rotate two eccentric reverse rotation, because the eccentric block of equal mass, force is produced only a vertical direction. Data acquisition and transmission by the acceleration sensor, charge amplifier, data acquisition instrument to complete. Data processing is processed by computer and professional software. The process of the experiment is: Firstly, put the engine on the experiment bench; secondly, arrange the acceleration sensor and connect the test instrument; thirdly, test the Dynamic Stiffness and damping angel in different conditions; fourthly, record and deal with the data to draw required curves.

\section{Results of the Vibration System Experiment of Vehicle Engines}

In the Condition that Preload is $\mathbf{5 0 0} \mathbf{N}$ and Amplitude is $\mathbf{0 . 5 m m}$. The Curves of the dynamic stiffness and damping angel under that condition are shown in Figure 1 and 2. 


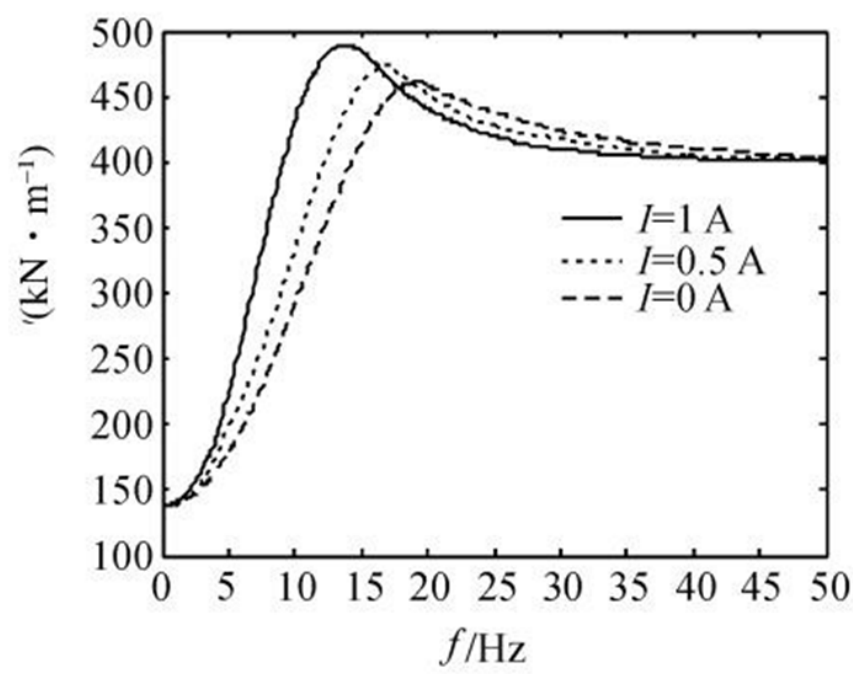

Figure 1 Curve of the dynamic stiffness under the condition that preload is $500 \mathrm{~N}$ and amplitude is $0.5 \mathrm{~mm}$

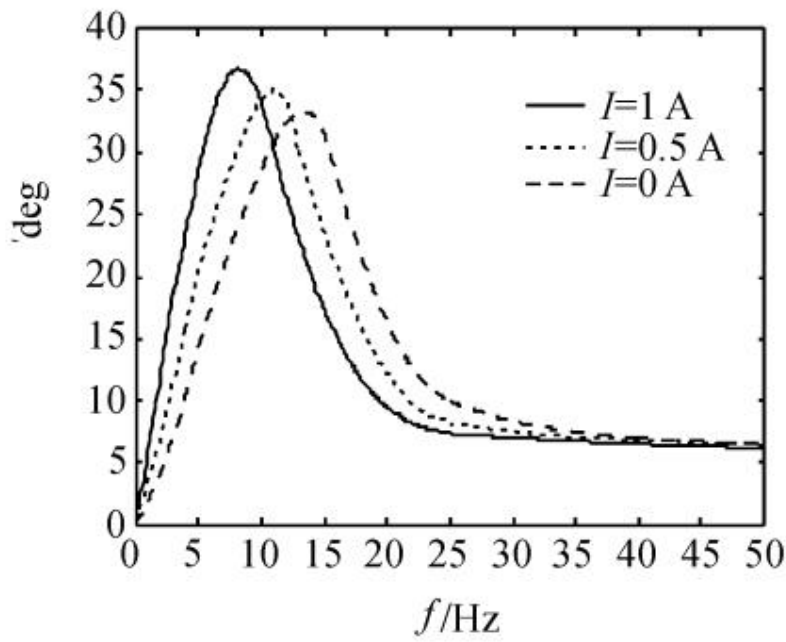

Figure 2 Curve of the damping angel under the condition that preload is $500 \mathrm{~N}$ and amplitude is $0.5 \mathrm{~mm}$

In the Condition that Preload is $\mathbf{5 0 0} \mathbf{N}$ and amplitude is $\mathbf{0 . 2 5} \mathrm{mm}$. The Curves of the dynamic stiffness and damping angel under that condition are shown in Figure 3 and 4.

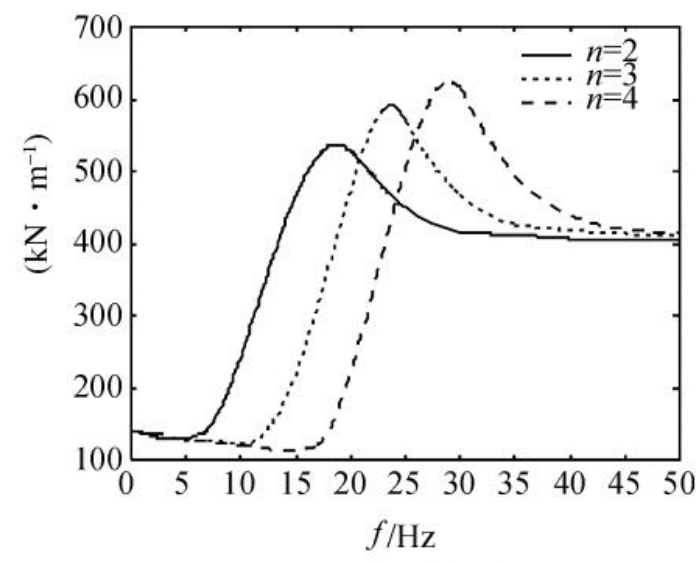

Figure 3 Curve of the dynamic stiffness under the condition that preload is $500 \mathrm{~N}$ and amplitude is $0.25 \mathrm{~mm}$ 


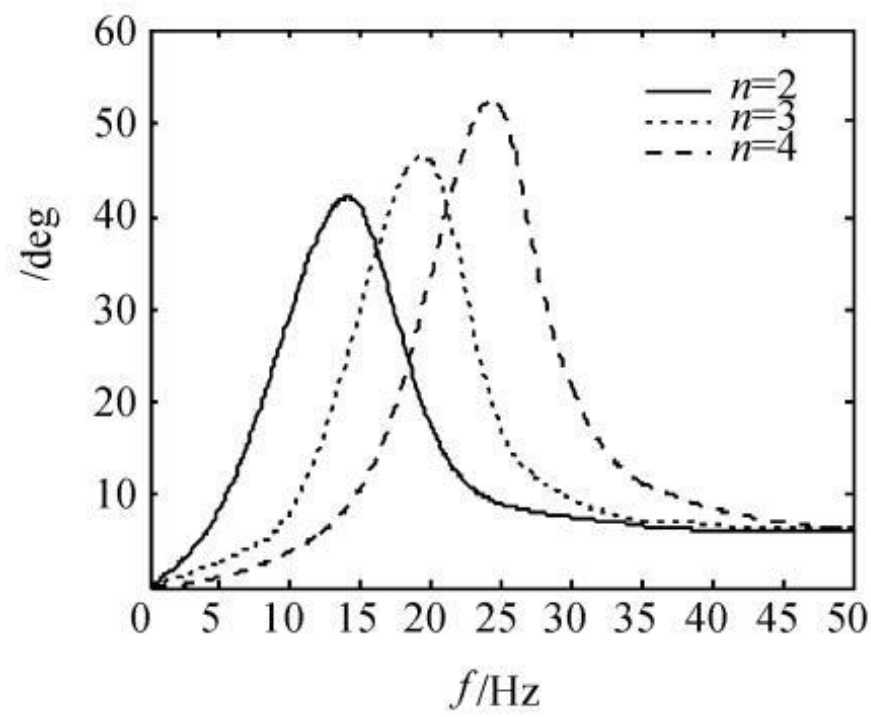

Figure 4 Curve of the damping angel under the condition that preload is $500 \mathrm{~N}$ and amplitude is

\section{$0.25 \mathrm{~mm}$}

Result Analysis. In general, at the range of $0-35 \mathrm{~Hz}$, the magneto rheological suspension dynamic stiffness and hysteresis angle have obvious nonlinear characteristics. The dynamic stiffness reaches the peak at about $15 \mathrm{~Hz}$; the damping angel reaches the peak at about $12 \mathrm{~Hz}$ lag angle. With the applied current becomes larger, the peak frequency slightly decreases. It proves the correctness of the modeling theory of the single channel magneto rheological suspension inertia.

\section{Conclusion}

This paper focuses on the dynamic characteristic analysis of the magneto rheological hydraulic suspension based on the experimental study. We first introduce the basic principle and method of dynamic characteristic test, prepare the test equipment and test samples, and analyze the characteristics of suspension dynamic simulation results, which verifies the accuracy of the simulation model.

\section{Acknowledgements}

This research is the results of the "Scientific Research Project in Sichuan Province Department of Education”. The Project Number is 15ZB0400.

\section{References}

[1] Liu Jing, Qin Wei, Fu Yuanlei, Zhu Bin, Auto Sci-tech, Vol. 39(2011) No2, p.37-40

[2] Zheng Ling, Deng Zhaoxue, Pang Jian, Xu Xiaomin, Fu Jianghua, Chen Daijun, Automotive Engineering, Vol. 38(2016) No 2, p.221-228

[3] Li Chenlong, Shen Yufeng, Jing Dong, Mining \& Processing Equipment, Vol. 42(2014) No 3, p.25-29

[4] Pan Gongyu, Wang Qianqian, Yang Xin, Journal of Mechanical \& Electrical Engineering, Vol. 32(2015) No 6, p.750-756 